LBNL-62345

CBP Tech Note-370

January 18, 2007

\title{
Spreader Design for FERMI@Elettra Free Electron Laser
}

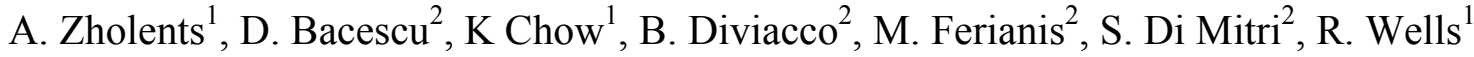 \\ 1) Lawrence Berkeley National Laboratory, Berkeley, CA 94720, USA \\ 2) Sincrotrone Trieste , 34012 Basovizza, Trieste, Italy
}

\begin{abstract}
In this note we describe a conceptual design of a part of the electron beam delivery system for FERMI@Elettra free electron laser (FEL) located between the end of the linac and the entrance to the FEL. This part includes the emittance diagnostic section, the electron beam switchyard for two FELs called spreader and matching sections. The design meets various constrains imposed by the existing and planned building boundaries, desire for utilization of existing equipment and demands for various diagnostic instruments.
\end{abstract}




\section{Introduction}

Two Free Electron Lasers (FEL1 and FEL2) are planed for a construction in the FERMI@Elettra project [1]. One linac will supply these FELs with the electron beam and only one FEL will operate at any given time. We call a spreader a part of the machine that directs the electron beam either into the FEL1 or into the FEL2. The spreader is connected to the linac with the emittance diagnostic section and to FEL1 and FEL2 with two matching sections for lattice functions. In this note we describe a design of the spreader and its adjacent sections.

\section{Spreader Lattice}

A simplified schematic of the spreader is shown in Figure 1. The spreader starts with two bending magnets with the positive polarity that turn the electron beam to the left. Then two bending magnets with the negative polarity turn the electron beam back into the straight line leading to FEL2 or, in the second case, the third magnet is switched off and the electron beam proceeds to the second pair of the magnets with the negative polarity that turn the electron beam back into the straight line leading to the FEL1. The design requirement is to shift FEL2 line by $1 \mathrm{~m}$ and FEL1 line by $3 \mathrm{~m}$ from the linac line.

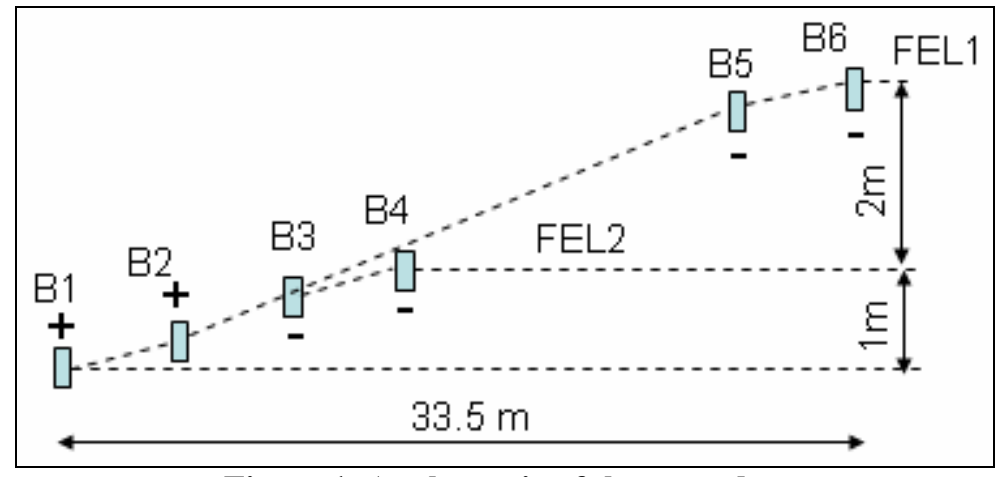

Figure 1. A schematic of the spreader.

A more detailed schematic of the part of the spreader leading to the FEL2 and Twiss functions inside this part are shown in Figure 2. Besides four bending magnets, it includes twelve quadrupoles that are arranged into a FODO lattice with $\pi / 2$ betatron phase advance in $\mathrm{x}$ and $\mathrm{y}$ planes per unit cell comprised of the two quadrupoles and two drifts. Two cells are used between each two adjacent bending magnets producing $-I$ transfer matrix in each plane. This arrangement in horizontal plane provides a basis for a compensation of emittance excitation due to coherent synchrotron radiation (CSR) [2,3] in the spreader's bending magnets. In order to show it, let us assume that the CSR in the B1 causes a change in energy for some electron on the amount of $\delta E$. Then defining the derivative of the dispersion function in the middle of the bending magnet as $D^{\prime}$, we obtain the kick to the electron trajectory at the end of the bending magnet:

$$
\Delta x^{\prime}=D^{\prime} \delta E
$$

and find an angle of the electron trajectory to be:

$$
x_{0}^{\prime}+D^{\prime} \delta E
$$


where $x_{0}^{\prime}$ is the angle of the electron trajectory without CSR. At the entrance of B2 after passing the lattice with $-I$ transport matrix this electron will have the angle:

$$
-x_{0}^{\prime}-D^{\prime} \delta E
$$

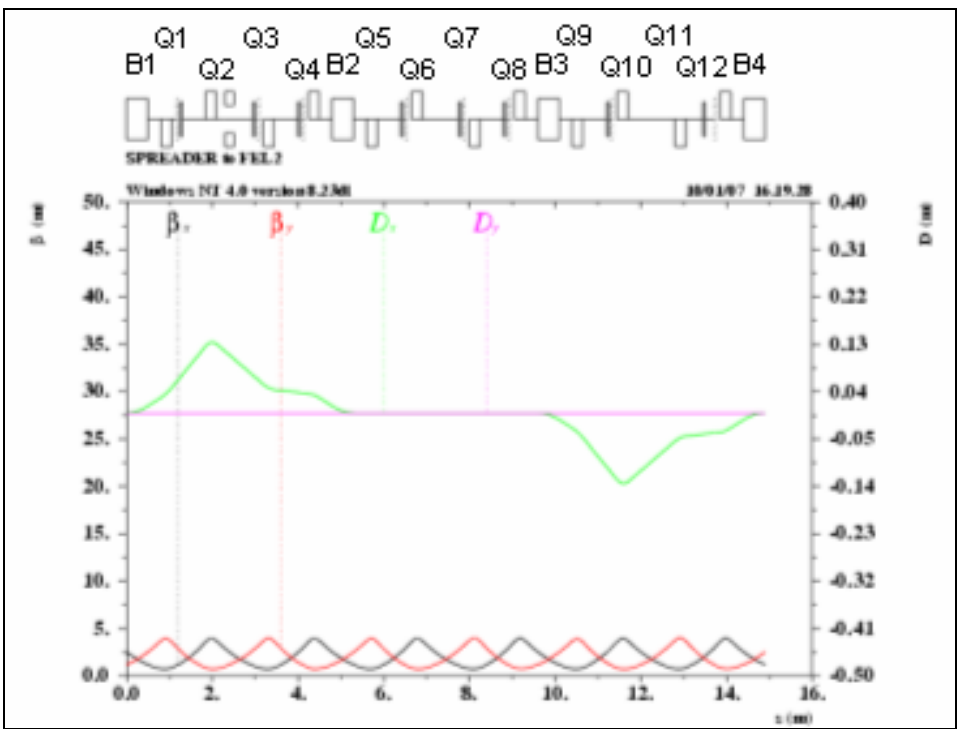

Figure 2. A schematic and lattice functions for a spreader part leading to FEL2. Black curve is the horizontal beta function, red curve is the vertical beta-function and green curve is dispersion function.

Now we assume that there is no mixing of the electron's longitudinal positions within the bunch on the way from B1 to B2. This ensures that the energy change experienced by the same electron in B2 due the CSR is exactly the same as in B1, i.e $\delta E$. Thus, because of this energy change and because of the fact that the derivative of the dispersion function in the middle of B2 is equal to $-D^{\prime}$ (see Figure 2), the angle of the electron trajectory emerging out of B2 will be:

$$
-x_{0}^{\prime}-D^{\prime} \delta E-D^{\prime} \delta E=-x_{0}^{\prime}-2 D^{\prime} \delta E
$$

After passing another section of the lattice between B2 and B3 with $-I$ transport matrix, the angle of the electron trajectory at the entrance of $\mathrm{B} 3$ will be:

$$
x_{0}^{\prime}+2 D^{\prime} \delta E
$$

Now assuming again the same energy change due to CSR and considering that the derivative of the dispersion function in the middle of B3 is equal to - $D^{\prime}$ (see, Figure 2), we find at the exit of B3:

$$
x_{0}^{\prime}+2 D^{\prime} \delta E-D^{\prime} \delta E=x_{0}^{\prime}+D^{\prime} \delta E
$$

After passing another section of the lattice between B3 and B4 with $-I$ transport matrix, the angle of the electron trajectory at the entrance of B4 will be:

$$
-x_{0}^{\prime}-D^{\prime} \delta E
$$

and because of the derivative of the dispersion function in the middle of B4 is equal to $D^{\prime}$ (see, Figure 2), the angle of the electron trajectory at the exit of B4 and a spreader will be:

$$
-x_{0}^{\prime}-D^{\prime} \delta E+D^{\prime} \delta E=-x_{0}^{\prime}
$$

Thus we proved that in the assumption of no mixing of longitudinal positions of electrons inside the electron bunch, i.e. in the assumption of a "frozen" electron density 
distribution, the spreader lattice designed according to the above described prescription should not increase the electron beam emittance due to the electron energy changes caused by CSR.

One of our goals is to have the spreader with a shortest possible length. Therefore we use there strong quadrupoles (see, Appendix A) and short drifts. Consequently, the bending angle for all four spreader's bending magnets is also predetermined as they must produce a $1 \mathrm{~m}$ off-set for the electron beam trajectory over the given length of the spreader.

The part of the spreader leading to FEL1 has a similar design, but has a larger distance between B2 and B5 (see, Figure 3) than the distance between B2 and B3 in spreader leading to FEL2. This was done in order to obtain a 3m off-set for FEL1 line beginning with the same pair of the bending magnets as in the FEL2 case. All quadrupoles shown in Figure 3 after B2 are different magnets than the ones in Figure 2 except Q8 which is used in both cases. Switching the operation from FEL2 to FEL1 will involve turning off B3, Q5, Q6, Q7 (shown in Figure 2) and re-tuning Q8 and few other quadrupoles in the emittance diagnostic section.

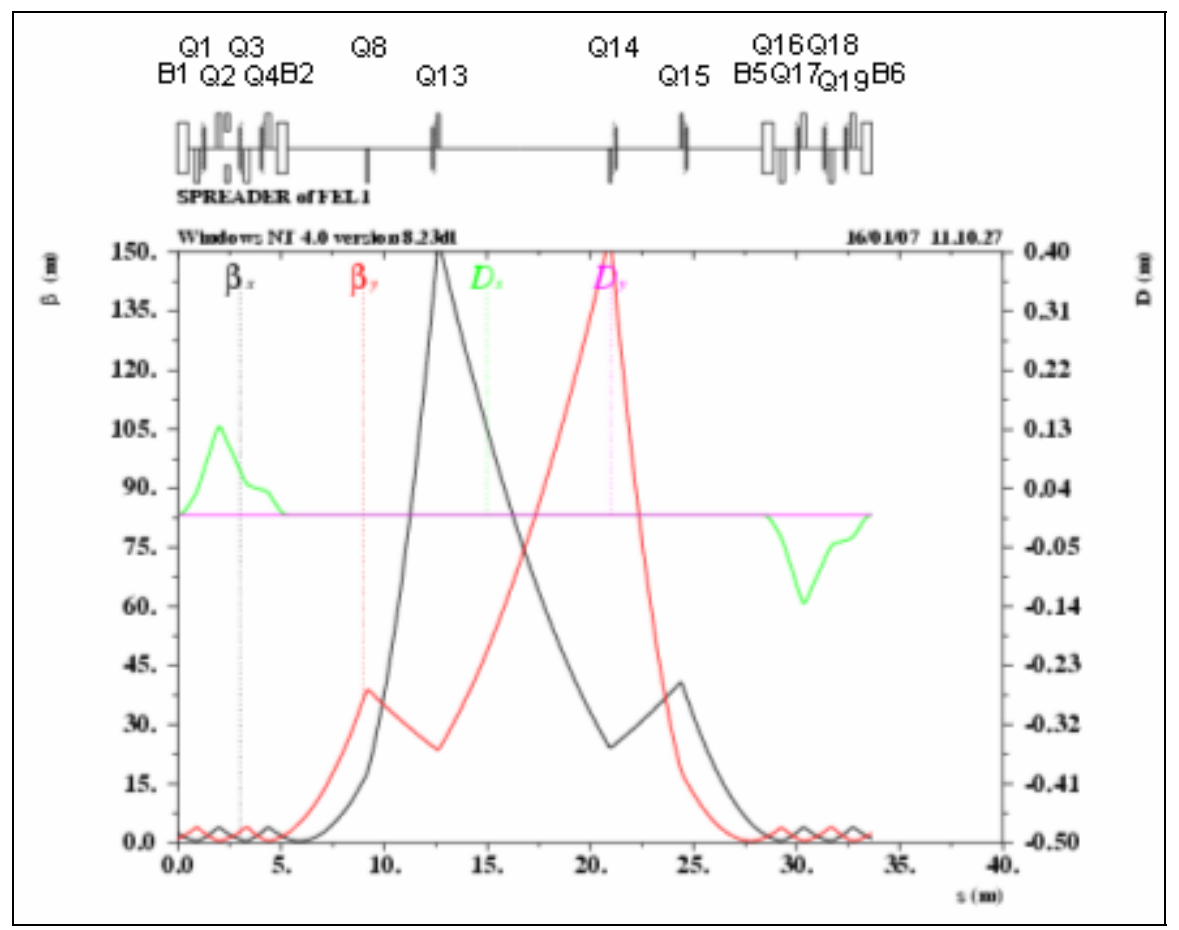

Figure 3. A schematic and lattice functions for a spreader part leading to FEL2: original lattice. Color coding is the same as in Figure 2.

Four quadrupoles in a region between $\mathrm{B} 2$ and $\mathrm{B} 5$ are spaced at larger distances than the quadrupoles in a region between $\mathrm{B} 1$ and $\mathrm{B} 2$ or $\mathrm{B} 5$ and $\mathrm{B} 6$. This produces larger beta functions in a region between $\mathrm{B} 2$ and $\mathrm{B} 5$. Part of that is also due to a mismatch of the beta functions between these three regions. In principle, one can add eight more quadrupoles in between B2 and B5 and have there a lattice with $3 \pi$ betatron phase advance and much smaller beta functions. This lattice will provide the same emittance compensation as the lattice with $\pi$ betatron phase advance. However we decided not to pursue this option (feared that adding more quadrupoles will create more interferences 
between FEL2 beam line and FEL1 beam line) and adapted the other approach to mitigate the problem. Namely we change the input Twiss functions at the entrance of the B1 and induce a small mismatch of the beta functions at the beginning with the beat waves of the beta functions that actually reduces the peak beta functions in the central part of the region between B2 and B5. This lattice is shown in Figure 4 and this is the one that will actually be using.

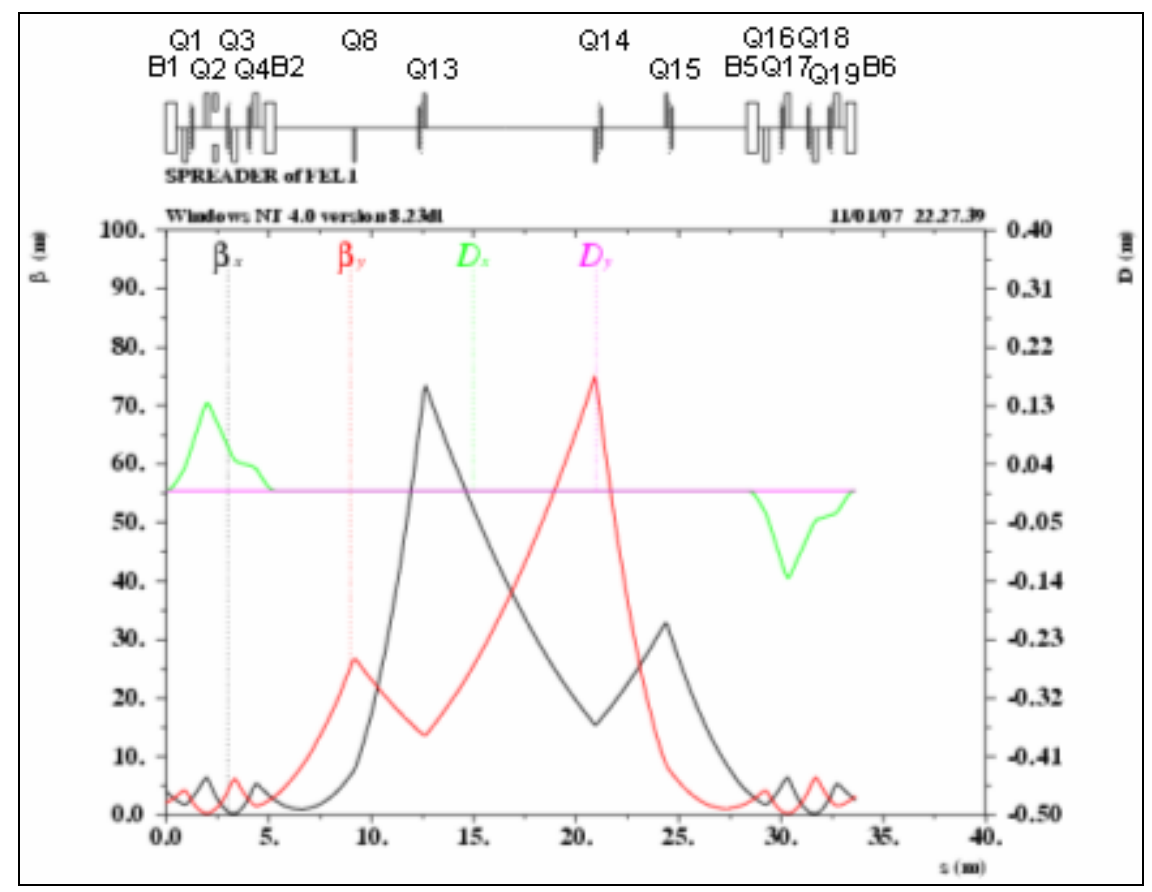

Figure 4. A schematic and lattice functions for a spreader part leading to FEL2: lattice with induced beating of beta functions. Color coding is the same as in Figure 2.

\section{Emittance Diagnostic Section}

Emittance diagnostic section is located between the linac and the spreader and is designed for emittance diagnostic using optical transition radiation from the screens that can be inserted into the electron beam path. Vertical and horizontal beam emittances will be deduced from the measurements of the electron beam sizes at several locations with various betatron phase advances [4]. This is the invasive technique and it will mainly be used at times when the machine does not operate for a production of the x-rays. Because of that we consider using two variants of the lattice: a) a production lattice optimized for a normal operation with a production of the x-rays and b) a diagnostic lattice optimized for emittance measurements. In the later case we use a periodic lattice with a betatron phase advancing from one screen to another screen by approximately $\pi / 4$ in both planes and with almost equal horizontal and vertical beta functions on all screens (see, Figure 5). The detailed information is given in Table 1. The RF deflecting cavity is located upstream of the screens and is going to be used for a slice emittance measurements. 


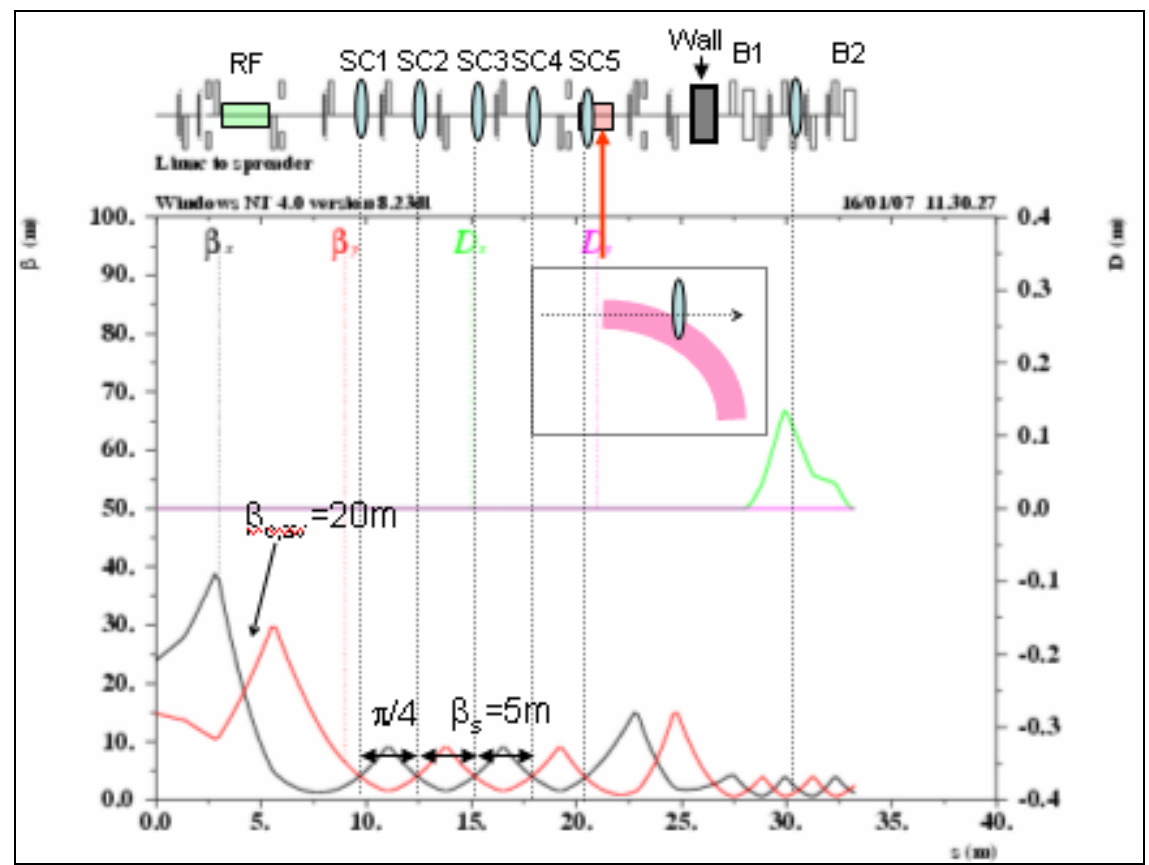

Figure 5. A schematic and lattice functions for the emittance diagnostic section beginning from the end of the linac and through the first two bending magnets of the spreader. Top line indicates the location of the rf deflecting cavity and screens. The insert shows the bending magnet for beam extraction into the regional dump. Wall separates the linac tunnel and the undulator hall.

Table 1. Twiss functions and betatron phase advance for deflecting cavity (DCAV) and screens in the emittance diagnostic section.

\begin{tabular}{|c|c|c|c|c|c|c|}
\hline $\begin{array}{l}\text { element } \\
\text { name }\end{array}$ & $\begin{array}{c}\mathrm{H} O \mathrm{R} \mathrm{I} \mathrm{Z} \\
\text { betax } \\
{[\mathrm{m}]}\end{array}$ & $\begin{array}{r}O N \mathrm{NA} \\
\text { alfax }\end{array}$ & $\stackrel{\operatorname{mux}}{[2 \mathrm{pi}]}$ & $\begin{array}{c}\mathrm{V} \text { E R T } \\
\text { betay } \\
{[\mathrm{m}]}\end{array}$ & $\begin{aligned} \text { I C A L } & \text { alfay } \\
& \end{aligned}$ & $\begin{array}{c}\text { muy } \\
{[2 \mathrm{pi}]}\end{array}$ \\
\hline DCAV & 17.848 & 6.448 & 0.024 & 19.041 & -3.773 & 0.051 \\
\hline SCREEN.1 & 4.337 & -1.503 & 0.372 & 3.938 & 1.396 & 0.123 \\
\hline SCREEN. 2 & 3.948 & 1.407 & 0.442 & 4.335 & -1.502 & 0.303 \\
\hline SCREEN.3 & 4.302 & -1.494 & 0.622 & 3.947 & 1.406 & 0.373 \\
\hline SCREEN. 4 & 3.936 & 1.395 & 0.692 & 4.304 & -1.495 & 0.553 \\
\hline SCREEN. 5 & 4.685 & -1.661 & 0.868 & 3.132 & 1.482 & 0.628 \\
\hline
\end{tabular}

The design was constrained by the location of the existing bending magnet that will remain in place and will be reused for the purpose of taking the electron beam into the region dump during commissioning and machine studies. This bending magnet can also serve for the beam abort from the FELs without affecting the linac operation. This side beam line will be equipped with additional screen that can be used for measurement of the slice energy spread (not designed at the moment). Another constrain was the location of the wall separating the linac tunnel and undulator hall. One of the design goals was to begin the spreader right after this wall at the beginning of the undulator hall and penetrate the wall just by the vacuum chamber such as to avoid interference with magnetic elements of the beam line. 


\section{Production Lattice}

The production lattice is shown in Figure 6. It is less favorable for emittance diagnostic because of the less periodic behaviour of Twiss function within the screen area and less regular advance of the betatron phase. But all screens can still be used for a quick beam characterization which is convenient when retuning of the quadrupoles for a transition into the diagnostics option of the lattice is not desirable. The detailed information for the production lattice is given in Table 2 .

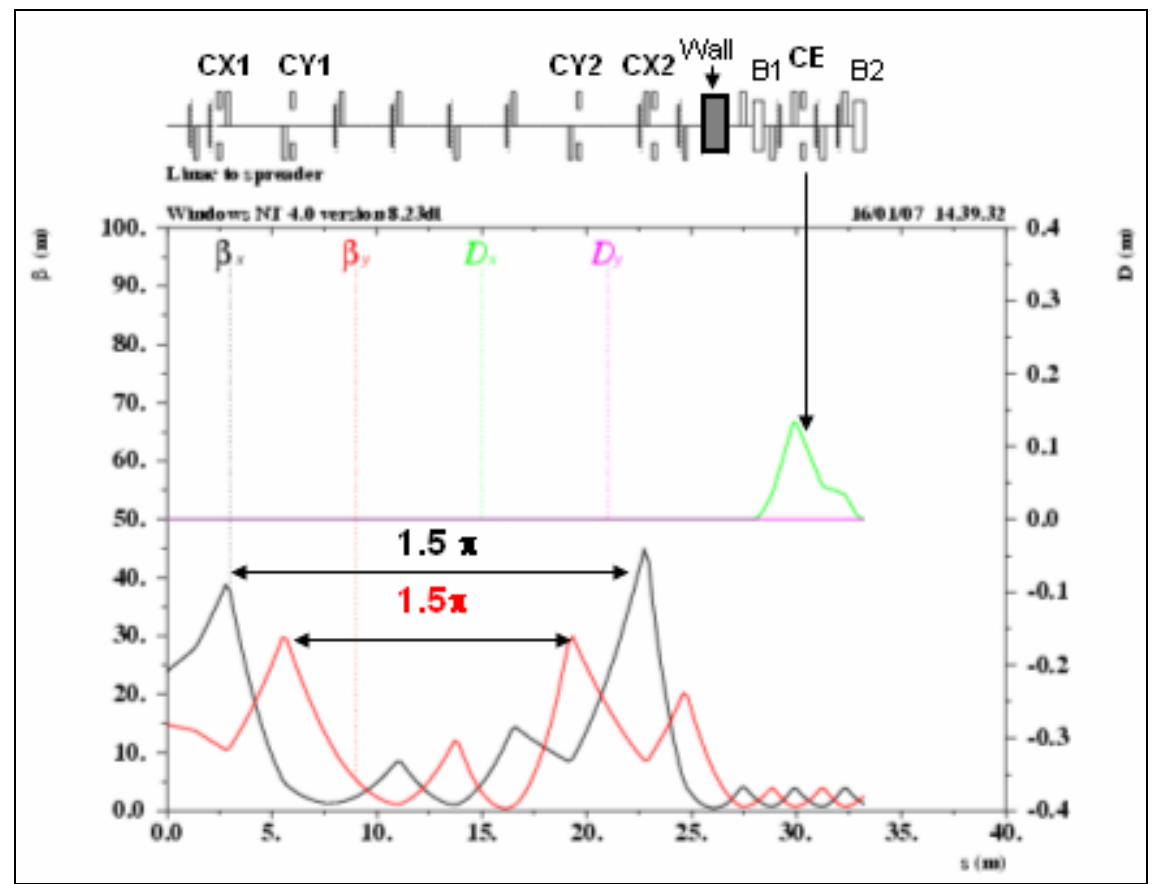

Figure 6. A schematic and lattice functions for the production lattice for FEL2 beginning from the end of the linac and through the first two bending magnets of the spreader. Top line indicates the location of collimators.

Table 2. Twiss functions and betatron phase advance for deflecting cavity (DCAV) and screens in the emittance diagnostic section for a case of the production lattice.

\begin{tabular}{|c|c|c|c|c|c|c|}
\hline $\begin{array}{l}\text { element } \\
\text { name }\end{array}$ & $\begin{array}{c}\text { O R I Z } \\
\text { betax } \\
{[\mathrm{m}]}\end{array}$ & $\begin{array}{l}\text { O N T A L } \\
\quad \text { alfax } \\
\end{array}$ & $\begin{array}{c}\operatorname{mux} \\
{[2 \mathrm{pi}]}\end{array}$ & $\begin{array}{l}\mathrm{V} E \mathrm{R} T \\
\text { betay } \\
{[\mathrm{m}]}\end{array}$ & $\begin{array}{l}\text { I C A I } \\
\text { alfay }\end{array}$ & $\begin{array}{c}\text { muy } \\
{[2 \mathrm{pi}]}\end{array}$ \\
\hline DCAV & 17.848 & 6.448 & 0.024 & 19.041 & -3.773 & 0.051 \\
\hline SCREEN. 1 & 4.131 & -1.394 & 0.364 & 2.948 & 1.261 & 0.134 \\
\hline SCREEN.2 & 3.003 & 1.386 & 0.442 & 5.094 & -2.193 & 0.358 \\
\hline SCREEN.3 & 5.667 & -2.589 & 0.674 & 2.274 & 2.035 & 0.427 \\
\hline SCREEN. 4 & 10.996 & 1.145 & 0.714 & 10.697 & -5.695 & 0.802 \\
\hline SCREEN. 5 & 19.616 & -4.813 & 0.754 & 19.944 & 3.354 & 0.823 \\
\hline
\end{tabular}

A distinctive feature of the production lattice is the presence of the two local peaks of the beta functions just upstream of the spreader. The lattice is tuned such as to have $3 / 2 \pi$ betatron phase advance between the local peaks of the beta functions where adjustable $\mathrm{x}$ 
and y collimators will be used. The energy collimator will be used near the peak of the dispersion function inside the spreader (see, Figure 6).

The production lattice for FEL1 shown in Figure 7 is slightly different, but it is only noticeable at the end of the emittance diagnostic section after B1. This difference is due to the introduced mismatch of the Twiss functions discussed previously.

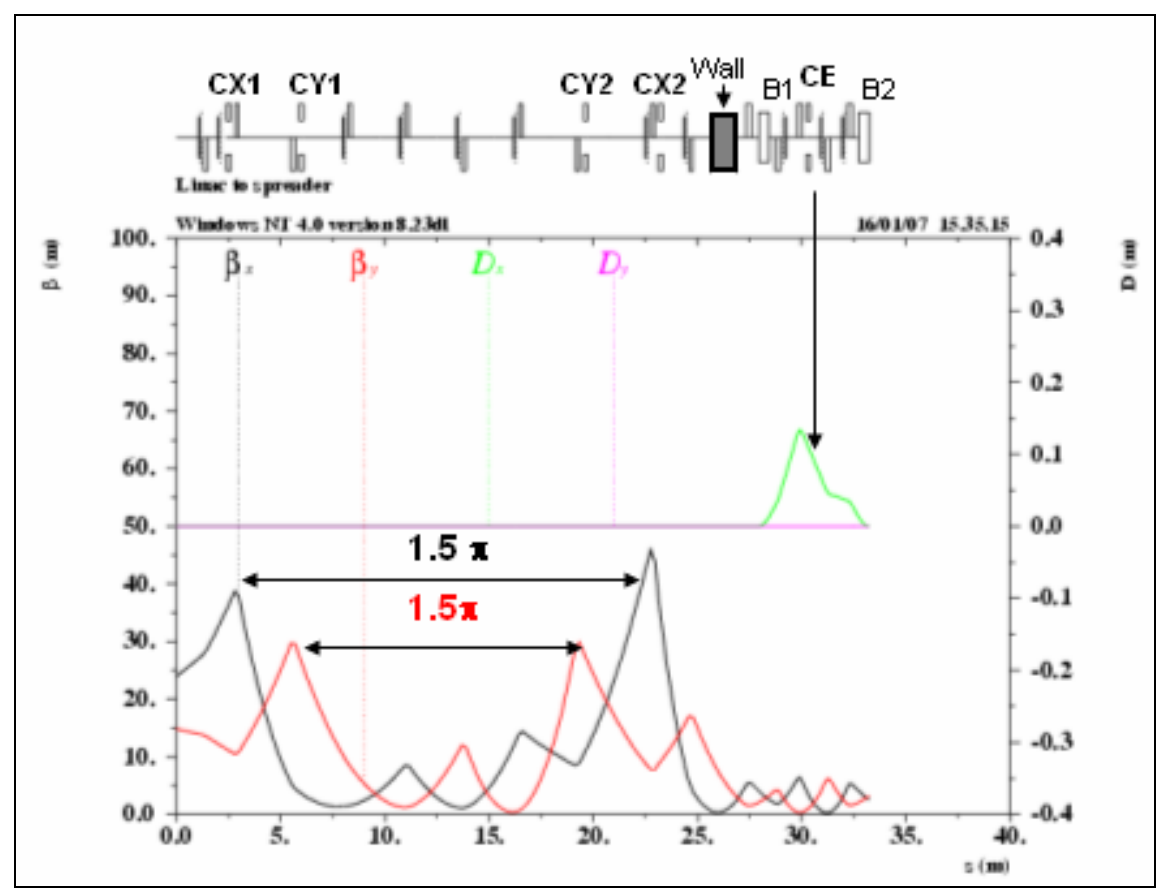

Figure 7. A schematic and lattice functions for the production lattice for FEL1 beginning from the end of the linac and through the first two bending magnets of the spreader. Top line indicates the location of collimators.

\section{Matching Sections after the Spreader}

Matching sections after the spreader are very similar for FEL2 and FEL1. Four quadrupoles are used for matching that provides enough flexibility to adjust Twiss function at the FEL input. A $5 \mathrm{~m}$ long drift is reserved after the last quadrupole and precision cavity BPMs will be placed on both sides of this drift. The electro-optical monitor for measurement of the electron bunch arrival time will be used inside this drift too. We note that if less than $5 \mathrm{~m}$ drift will be needed, this can be easily accommodated by a slight re-adjustment of the quadrupoles strengths. The entire lattice for FEL1 beginning from the end of the linac and including matching sections is shown in Figure 8.

\section{Other Equipment}

Horizontal and vertical steering magnets and beam position monitors have been placed throughout the lattice. They can be seen positioned next to almost every quadrupole magnet in the layout plots that will be described in the next section. 


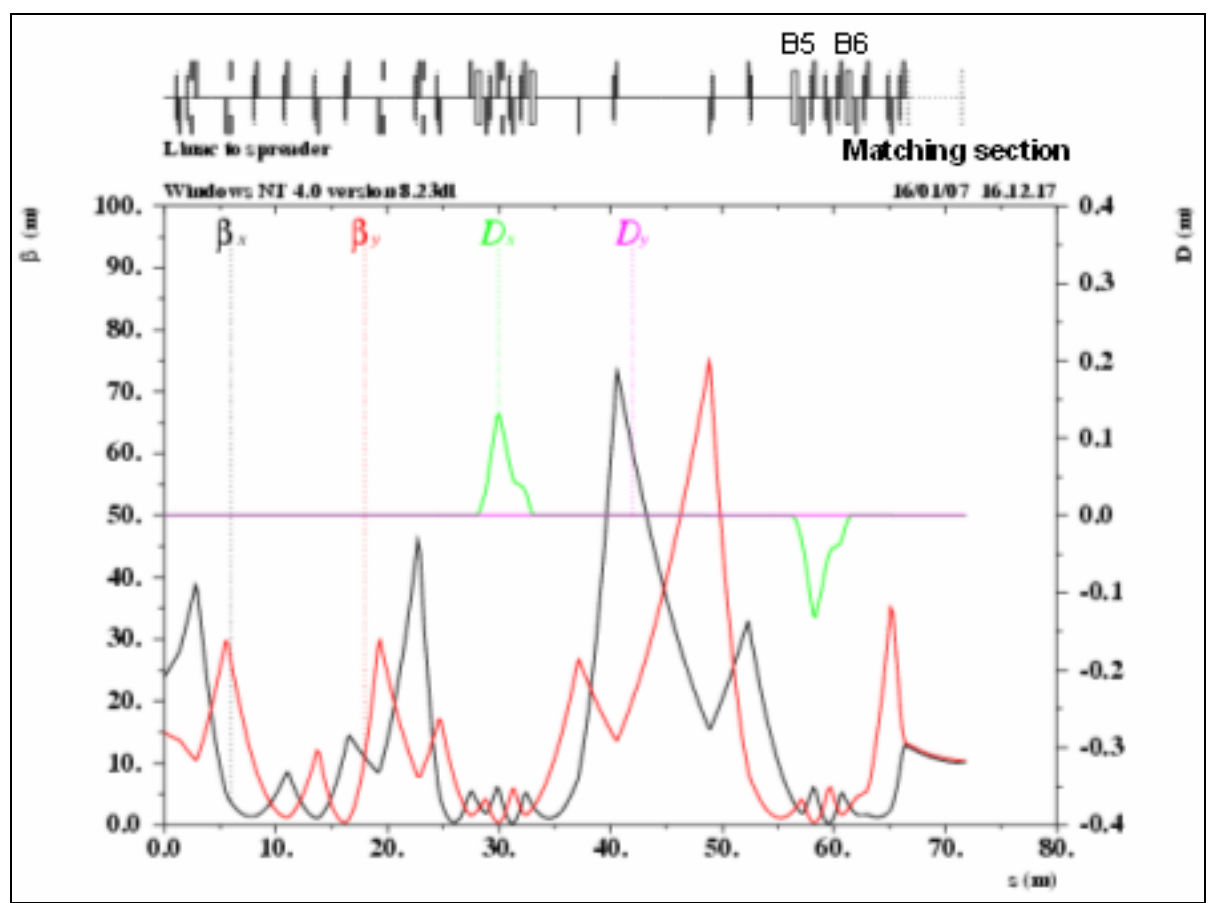

Figure 8. Twiss functions for the production lattice for FEL1 beginning from the end of the linac and ending at the beginning of the FEL1. Matching section is located after B6.

\section{Time-off-flight adjustment}

The time-off-flight parameter $R_{56}$ of the spreader shown in Figures 2 or Figure 4 is equal to $0.9 \mathrm{~mm}$. We show here that it can be adjusted in a relatively large range of values. In order to do this one needs to tune synchronously the quadrupole Q2 located near the peak of the dispersion function and the quadrupole Q10 in the case of the FEL2 spreader part (or quadrupole Q17 in the case of FEL1 spreader part). Then the dispersion function gets a kick at Q2, oscillates between B2 and B3 magnets and gets a compensating kick at Q10 (or Q17). Figure 9 shows the spreader part for FEL2 when both quadrupoles Q2 and Q10 were tuned down by $10 \%$ in order to increase $R_{56}$ to 5.5 $\mathrm{mm}$. Such adjustments for $R_{56}$ were previously considered in [5] for dissolving of the clusters of electrons at the edges of the electron bunch. As it is seen in Figure 9, this adjustment causes some beating of the beta-functions at the end of the lattice that must be corrected in the matching lattice downstream.

Tweaking the same quadrupoles in the opposite directions, i.e. reducing the gradient in one quadrupole and increasing in the another quadrupole by the same amount, allows fine adjustments of the dispersion function leaking out of the spreader due to various errors without affecting the beta-functions and $R_{56}$. This example is shown in Figure 10. 


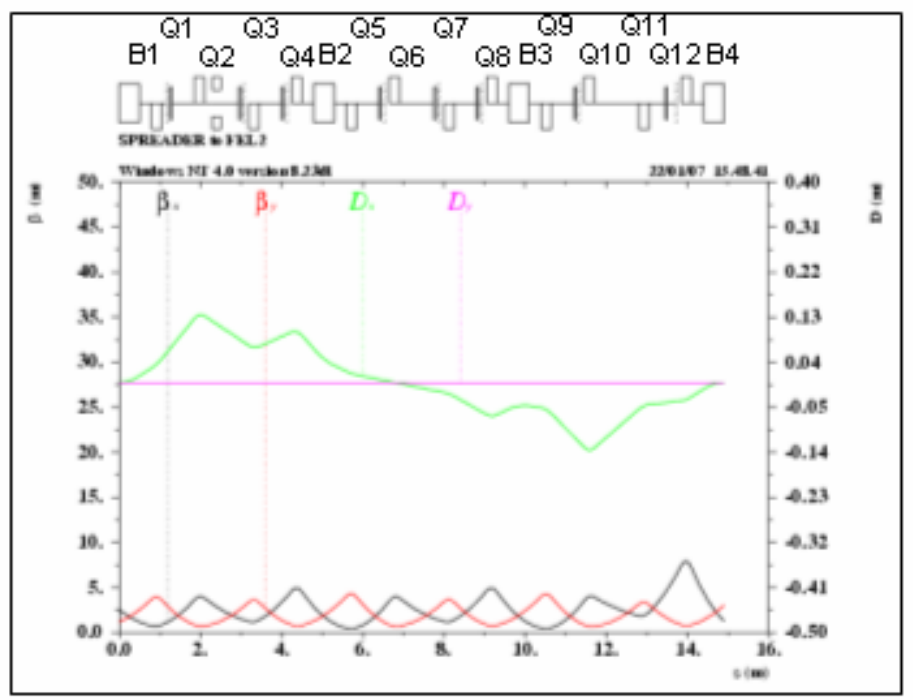

Figure 9. A schematic and lattice functions for a spreader part leading to FEL2. Reduction of the gradiens in the quadrupoles Q2 and Q10 by $10 \%$ changed $R_{56}$ from $0.9 \mathrm{~mm}$ to $5.5 \mathrm{~mm}$.

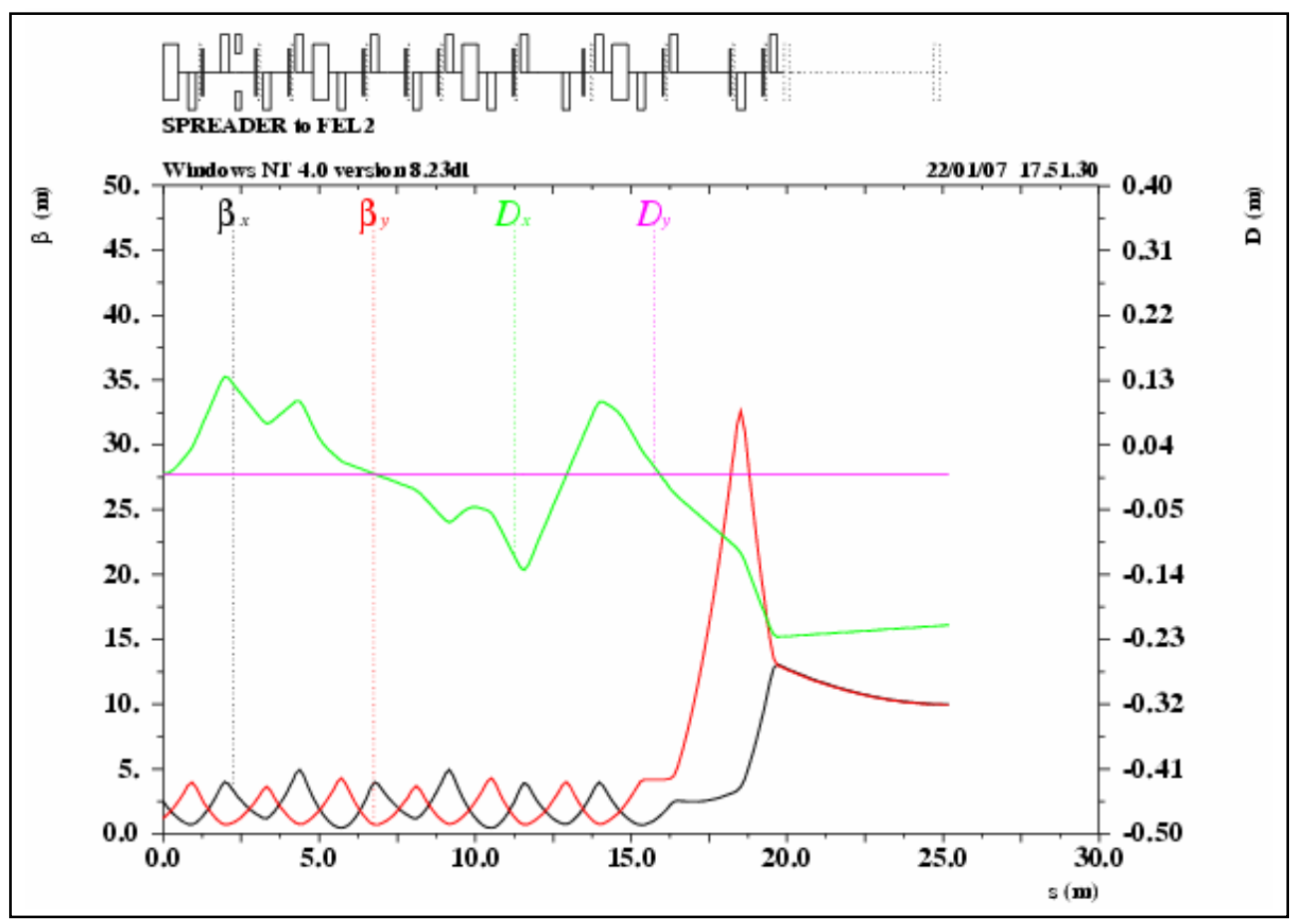

Figure 10. Dispersion after the spreader is created by $10 \%$ reduction of the gradient in $Q 2$ and $10 \%$ increase of the gradient in Q10.

\section{Layout Details}

The drawing with the spreader layout is too large to be included into this note and is given in a supplemental file called "spreader_layout.pdf" [6]. Here we only show two 
most difficult areas with a high congestion of various apparatus. Figure 11 shows the layout of the area downstream of B3 bending magnet where two beam lines split.

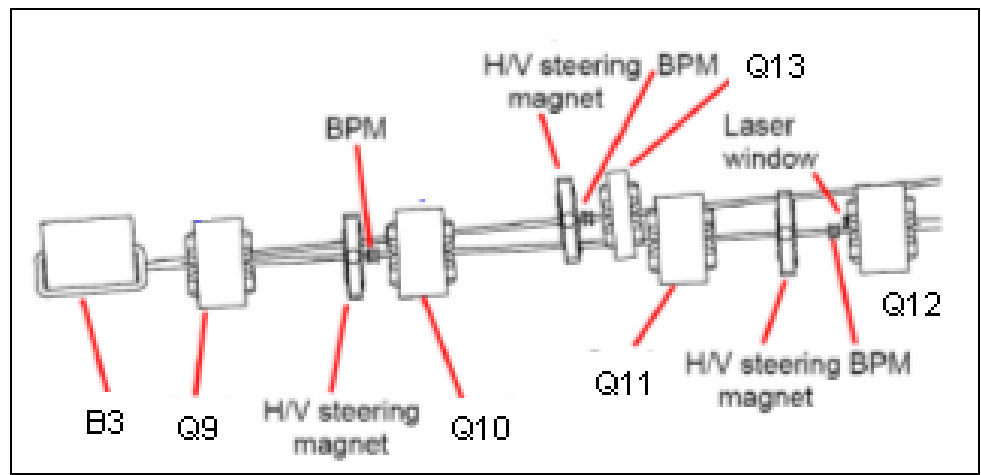

Figure 11. The layout of the area after B3 bending magnet where two beam lines split.

The electron beam following to FEL2 goes through the centers of the quadrupoles Q9, Q10, Q11 and Q12, and the electron beam following to FEL1 goes through the center of the quadrupole Q13. In the cases of Q10, Q11, Q12 and Q13 quadrupoles, the periphery beam passes the quadrupole rather far from the center of the quadrupoles without causing much of the interference. But in the case of quadrupole Q9, the periphery beam is located still within the overall dimensions of the quadrupole (see, Figure 12) which means that this quadrupole must accommodate the vacuum chamber for both beams. The location of this quadrupole is chosen such as to make it feasible and a conceptual design of the quadrupole is shown in Figure 12. But a detailed design of this quadrupole must be further pursued.

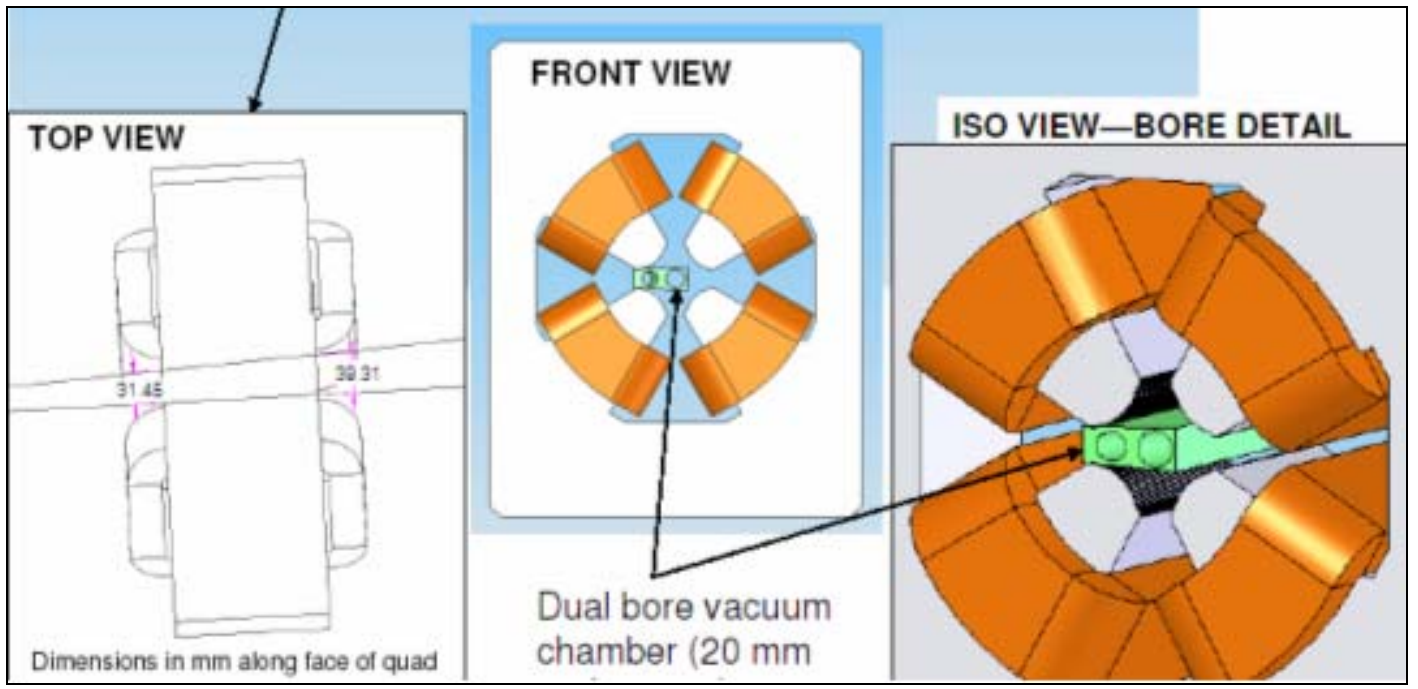

Figure 12. Top, front and isometric view of the quadrupole Q9 showing also electron beam trajectories and a sketch of the vacuum chambers for the central and periphery beams.

Figure 13 shows the seed laser port and surrounding apparatus. The location for this port just upstream of the last bending magnet B4 is the closest to FEL2 and it is still more than $11 \mathrm{~m}$ away from the FEL2. A part of it is due to the long drift space taken by various diagnostics upstream of the FEL2. Figure 14 shows fine details for the laser port such as 
flange and widow sizes and window separation from the electron beam axis. Similar to the quadrupole Q9, the quadrupole Q12 accommodates the vacuum chamber that allows two beams, the electron beam and the laser beam, both heading to the FEL2.

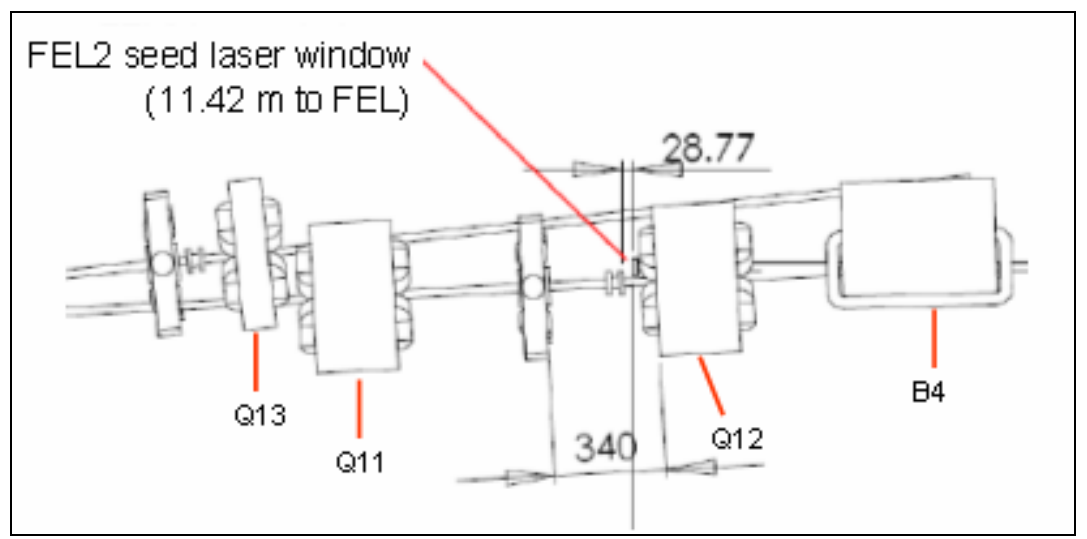

Figure 13. Top view on the seed laser port for FEL2 and surrounding apparatus.

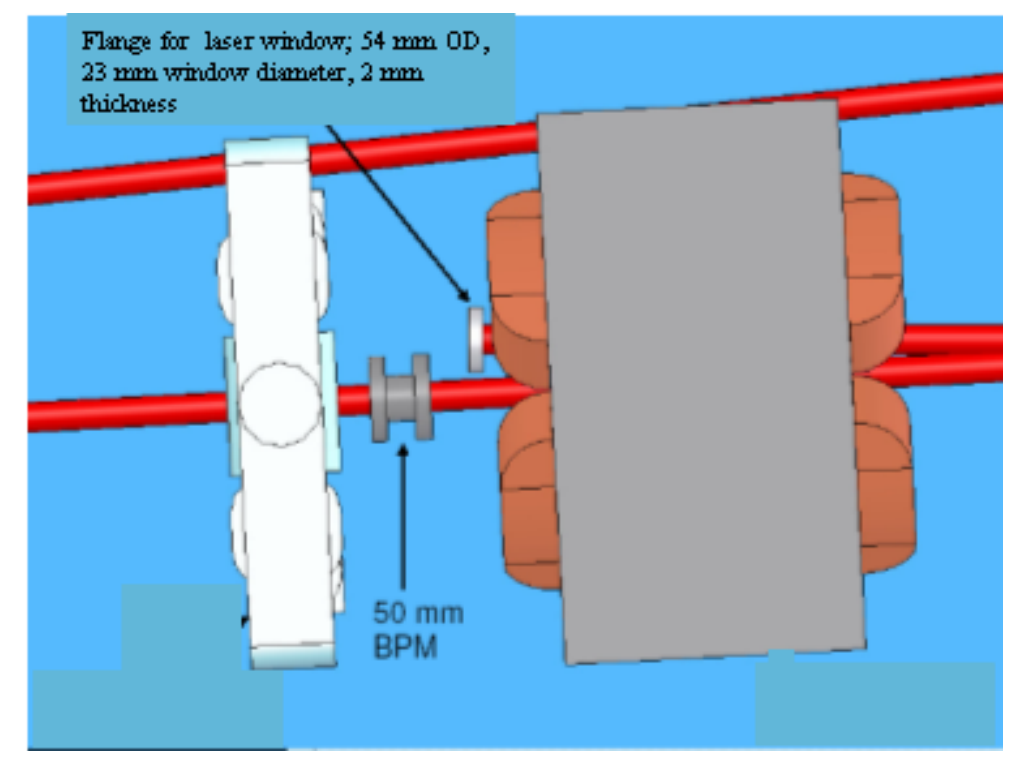

Figure 14. Fine details for the seed laser port.

The design of the seed laser port for FEL1 is exactly the same as just described design of the seed laser port for FEL2. This port is located upstream of the bending magnet B6 and quadrupole Q18.

\section{Other Ideas}

Other ideas that we explored during the design phase and then abounded include a concept when the entire emittance diagnostic section is placed in a straight line downstream of the first spreader magnet B1 at some distance to avoid the interference with the spreader. Theoretically this allows shorter lattice, but when we actually designed it, we found that we only shortened the lattice by approximately $8 \mathrm{~m}$. The downside of 
this approach was that the spreader was moved upstream and into the linac tunnel and significantly interfered with the wall separating the tunnel and the undulator hall.

Another exploratory study was the attempt to find the lattice that can combine emittance diagnostic and collimation provisions without the need to switch from one lattice to another lattice. We found that it was not possible to do within the existing boundaries and that we actually need to expanded the lattice at least by two more quadrupoles and move the emittance diagnostic section well beyond the wall separating the linac tunnel and undulator hall.

\section{Particle Tracking}

As mentioned in Paragraph 7 of this note, reference [6] contains the engineering efforts performed toward a technical realization of the spreader layout. In May 2006 the layout of a four branch spreader for FERMI@Elettra was explored. That layout reflected the two branch spreader layout presently developed but with mirror symmetry in the bending plane with respect to the linac axis. Therefore the electron bunch obtained a total lateral deflection by four dipole magnets for each beam line, with a bending angle of $3.25^{\circ}$ per dipole ( $3^{\circ}$ per dipole is in the present layout). The compensation of the CSR emittance growth by means of the $-I$ transport matrix was implemented in the four branch spreader.

Particle tracking through the two versions of the spreader layout has been performed in order to check important differences, if any, in the beam dynamics in presence of CSR. Such a different dynamics could be addressed, in principle, to the different bending angle per beam line in the two layouts and to the $2^{\text {nd }}$ order geometric and chromatic aberrations in presence of different quadrupole magnets strengths. In particular, the production lattice of the two branch spreader layout foresees higher $\beta$-functions designed for the collimation process. Tracking results have been obtained with Elegant [7]; they are shown in Figure 13 for the Medium Bunch Length (MBL) mode and in Figure 14 for the Long Bunch (LB) mode [1]. Table 3 lists the main beam parameters for the two bunch options.

Table 3. Main electron beam parameters of the MBL and LB mode.

\begin{tabular}{|l|c|c|c|}
\hline & MEDIUM & LONG & Units \\
\hline Bunch Charge & 0.80 & 1.00 & $\mathrm{nC}$ \\
\hline Bunch Length, FWHM & 700 & 1400 & $\mathrm{fs}$ \\
\hline Peak Current & 800 & 500 & $\mathrm{~A}$ \\
\hline Slice Norm. Emittance & 1.5 & 1.5 & $\mu \mathrm{m}$ \\
\hline Total Energy Spread, RMS & 0.07 & 0.05 & $\%$ \\
\hline
\end{tabular}



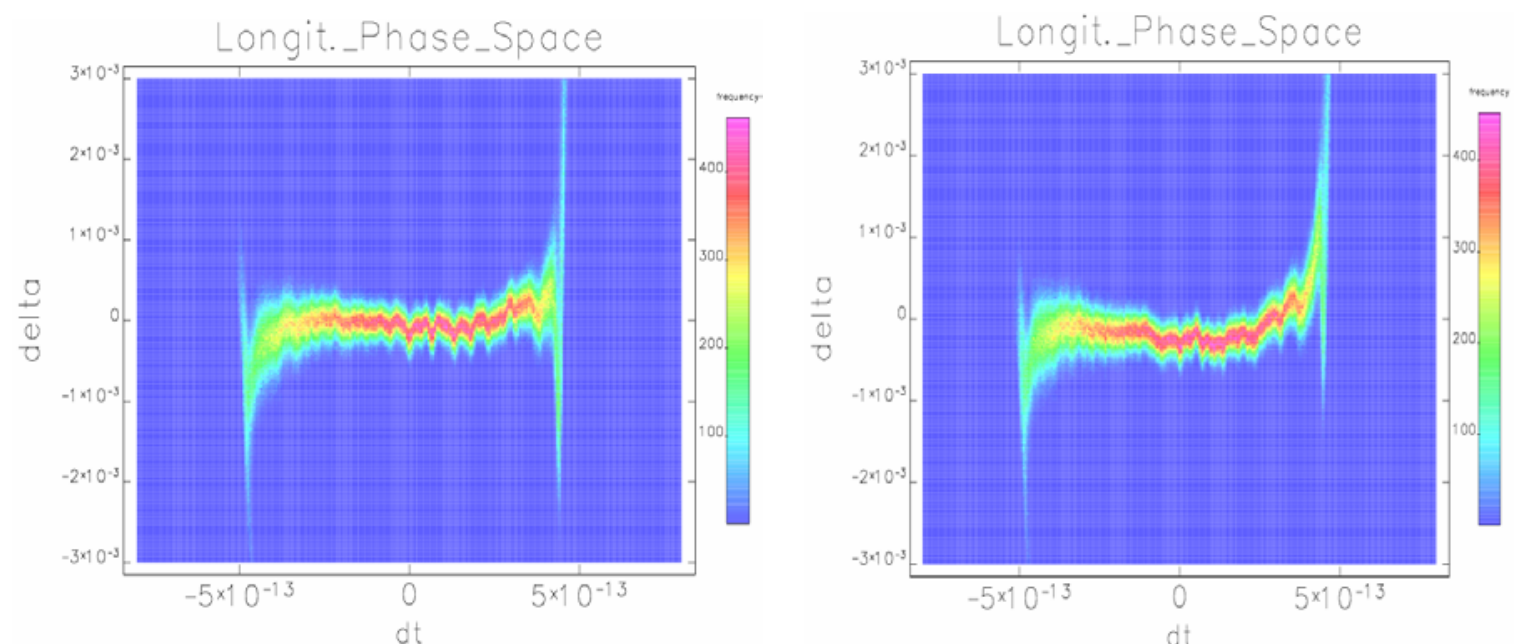

Figure 13. Longitudinal phase space (relative energy spread vs. bunch duration) at the spreader end for the MBL mode produced by Elegant tracking with 1 million macroparticles. $2^{\text {nd }}$ order beam transport and 1-D model for CSR in the spreader dipoles are included. Left plot: tracking through the FEL1 beam line of the four branch spreader. Right plot: tracking through the FEL1 beam line of the two branch spreader (production lattice).
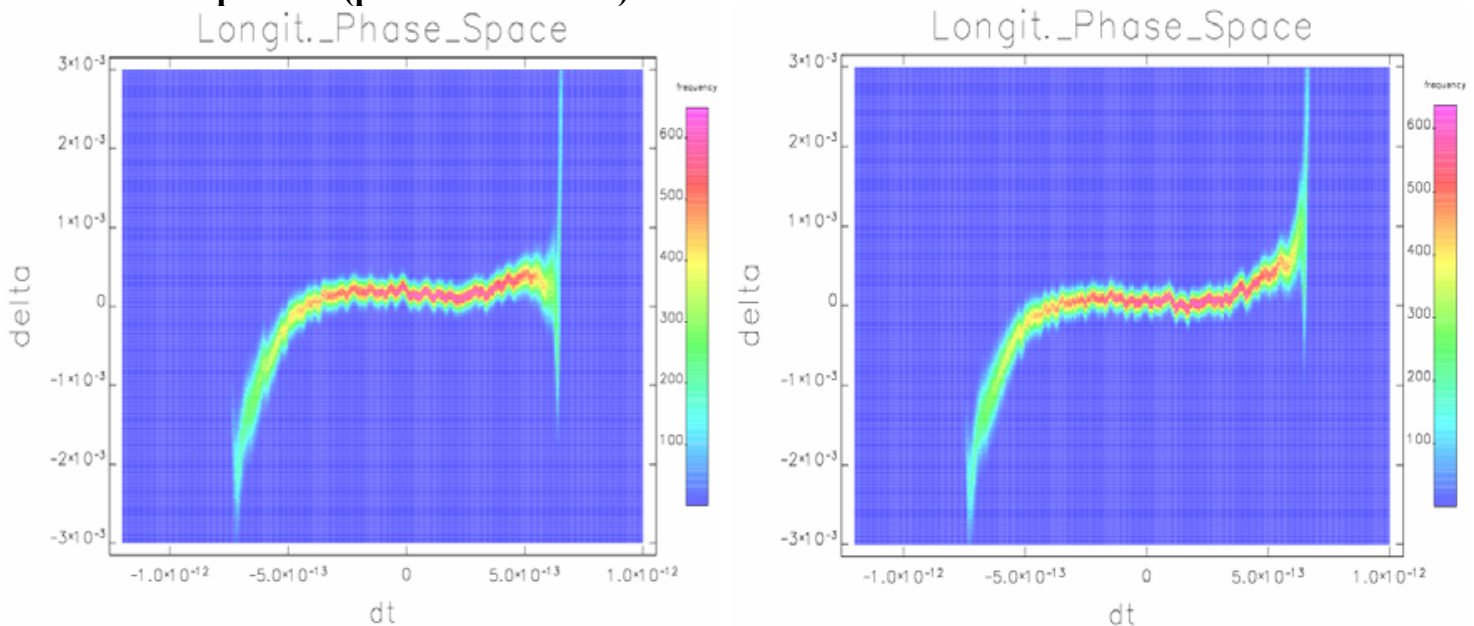

Figure 14. . Longitudinal phase space (relative energy spread vs. bunch duration) at the spreader end for the LB mode produced by Elegant tracking with 1 million macroparticles. $2^{\text {nd }}$ order beam transport and 1-D model for CSR in the spreader dipoles are included. Left plot: tracking through the FEL2 beam line of the four branch spreader. Right plot: tracking through the FEL2 beam line of the two branch spreader (production lattice).

By comparing the two adjacent plots in Figure 13 and in Figure 14, it is clearly visible that the longitudinal dynamics is the same in the four and in the two branch spreader layout, both for the MBL and LB mode. The same conclusion is reached as for the total transverse emittance behaviour: no emittance growth is observed in both the spreader layouts.

\section{Summary}

In conclusion, we completed a conceptual design of the spreader and adjacent section. This design meets physics requirements for emittance preservation, diagnostic demands, 
collimation and other constrains imposed by building boundaries and a desire for reutilization of the existing equipment.

\section{Appendix A}

Here we give the list of all quadrupoles used in operation for either FEL1 or FEL2 and show their gradients for $1.5 \mathrm{GeV}$ electron beam energy and for two mode of operation discussed in the main text, i.e. x-ray production and emittance diagnostics. We used 1.5 $\mathrm{GeV}$ rather than $1.2 \mathrm{GeV}$ to ensure an extra margin on the performance.

Mode of operation

NAME

"Q_EM.1"

"Q_EM.2"

"Q_EM.3"

"Q_EM.4"

"Q_EM.5"

Mode of operation

NAME

"Q_EM.6"

"Q_EM.7"

"Q_MT.1"

"Q_MT.2"

"Q_MT.3"

"Q_MT.4"

"Q_SPRD.1"

"Q_SPRD.2"

"Q_SPRD.3"

"Q_SPRD.4"

"Q_SPRD.8"

"Q_SPRD.13"

"Q_SPRD.14"

"Q_SPRD.15"

"Q_SPRD.16"

"Q_SPRD.18"

"Q_SPRD.18"

"Q_SPRD.19"

"Q_SPRD.20"

"Q_SPRD.21"

"Q_SPRD.22"

"Q_SPRD.23"

"Q_EM.1"

"Q_EM.2"

"Q_EM.3"

"Q_EM.4"

"Q_EM.5"

"Q_EM.6"

"Q_EM.7"

$$
\begin{array}{r}
\mathrm{S}(\mathrm{m}) \\
1.5
\end{array}
$$$$
\begin{array}{r}
1.5 \\
3
\end{array}
$$

5.7

8.43

11.16

$\mathrm{S}(\mathrm{m})$

13.89

16.62

19.35

22.95

24.81

27.59

28.99

30.04

31.39

32.44

37.165

40.615

48.94

52.39

57.34

58.39

59.74

60.79

62.14

63.19

65.34

66.39

1.5

3

5.7

8.43

11.16

13.89

16.62

$\mathrm{L}(\mathrm{m})$
0.2
0.2
0.2
0.2
0.2

$\mathrm{L}(\mathrm{m})$

0.2

0.2

0.2

0.2

0.2

0.25

0.25

0.25

0.25

0.25

0.1

0.1

0.1

0.1

0.25

0.25

0.25

0.25

0.25

0.25

0.25

0.25

0.2

0.2

0.2

0.2

0.2

0.2

0.2
FEL1 production

$\mathrm{K} 1 \mathrm{~L}(1 / \mathrm{m})$
$-6.74 \mathrm{E}-02$
0.353664
-0.34
$2.42 \mathrm{E}-02$
0.58

FEL2 production

$\mathrm{K} 1 \mathrm{~L}(1 / \mathrm{m})$ $-0.72$

0.4

$-0.4697$

0.547738

$-0.481248$

1.0398

$-1.2823$

1.2823

$-1.2823$

1.2823

$-0.265707$

0.265707

$-0.265707$

0.265707

$-1.2823$

1.2823

$-1.2823$

1.2823

$-0.29746$

0.607924

$-0.69322$

0.586954

$-6.74 \mathrm{E}-02$

0.353664

$-0.34$

2.42E-02

0.58

$-0.72$

0.4

$G(T / m)$
$-1.69 \mathrm{E}+00$
$8.85 \mathrm{E}+00$
$-8.51 \mathrm{E}+00$
$6.06 \mathrm{E}-01$
$1.45 \mathrm{E}+01$

$\mathrm{G}(\mathrm{T} / \mathrm{m})$

$-1.80 \mathrm{E}+01$

$1.00 \mathrm{E}+01$

$-1.18 \mathrm{E}+01$

1.37E+01

$-1.20 E+01$

$2.08 \mathrm{E}+01$

$-2.57 \mathrm{E}+01$

2.57E+01

$-2.57 \mathrm{E}+01$

2.57E+01

$-1.33 E+01$

1.33E+01

$-1.33 E+01$

$1.33 \mathrm{E}+01$

$-2.57 \mathrm{E}+01$

2.57E+01

$-2.57 E+01$

2.57E+01

$-5.95 \mathrm{E}+00$

1.22E+01

$-1.39 \mathrm{E}+01$

1.17E+01

$-1.69 E+00$

$8.85 \mathrm{E}+00$

$-8.51 E+00$

6.06E-01

$1.45 \mathrm{E}+01$

$-1.80 \mathrm{E}+01$

1.00E+01
FEL1 diagnostics

\begin{tabular}{cc}
$\mathrm{K} 1 \mathrm{~L}(1 / \mathrm{m})$ & \multicolumn{1}{c}{$\mathrm{G}(\mathrm{T} / \mathrm{m})$} \\
$-6.74 \mathrm{E}-02$ & $-\mathbf{1 . 6 9 E}+00$ \\
0.353664 & $\mathbf{8 . 8 5 E + 0 0}$ \\
-0.327239 & $\mathbf{- 8 . 1 9 E + 0 0}$ \\
$2.42 \mathrm{E}-02$ & $\mathbf{6 . 0 6 E}-01$ \\
0.531192 & $\mathbf{1 . 3 3 E + 0 1}$
\end{tabular}

FEL2 diagnostics$$
\mathrm{K} 1 \mathrm{~L}(1 / \mathrm{m})
$$

$-0.531192$

0.531192

$-0.446484$

0.619137

$-0.583121$

0.756301

$-1.2823$

1.2823

$-1.2823$

1.2823

$-0.265707$

0.265707

$-0.265707$

0.265707

$-1.2823$

1.2823

$-1.2823$

1.2823

$-0.29746$

0.607924

$-0.69322$

0.586954

$-6.74 \mathrm{E}-02$

0.353664

$-0.327239$

2.42E-02

0.531192

$-0.531192$

0.531192
$\mathrm{G}(\mathrm{T} / \mathrm{m})$

-1.33E+01

1.33E+01

$-1.12 E+01$

1.55E+01

$-1.46 E+01$

1.51E+01

$-2.57 \mathrm{E}+01$

2.57E+01

$-2.57 E+01$

2.57E+01

$-1.33 \mathrm{E}+01$

1.33E+01

$-1.33 E+01$

1.33E+01

$-2.57 E+01$

2.57E+01

$-2.57 E+01$

2.57E+01

$-5.95 \mathrm{E}+00$

1.22E+01

$-1.39 \mathrm{E}+01$

1.17E+01

$-1.69 \mathrm{E}+00$

$8.85 \mathrm{E}+00$

$-8.19 E+00$

6.06E-01

1.33E+01

$-1.33 E+01$

1.33E+01 


$\begin{array}{llrrrrr}\text { "Q_MT.1" } & 19.35 & 0.2 & -0.461362 & \mathbf{- 1 . 1 5 E + 0 1} & -0.583424 & \mathbf{- 1 . 4 6 E + 0 1} \\ \text { "Q_MT.2" } & 22.95 & 0.2 & 0.545362 & \mathbf{1 . 3 6 E + 0 1} & 0.586168 & \mathbf{1 . 4 7 E + 0 1} \\ \text { "Q_MT.3" } & 24.81 & 0.2 & -0.53367 & \mathbf{- 1 . 3 4 E + 0 1} & -0.727166 & \mathbf{- 1 . 8 2 E + 0 1} \\ \text { "Q_MT.4" } & 27.59 & 0.25 & 1.25405 & \mathbf{2 . 5 1 E + 0 1} & 0.845493 & \mathbf{1 . 6 9 E + 0 1} \\ \text { "Q_SPRD.1" } & 28.99 & 0.25 & -1.2823 & \mathbf{- 2 . 5 7 E + 0 1} & -1.2823 & \mathbf{- 2 . 5 7 E + 0 1} \\ \text { "Q_SPRD.2" } & 30.04 & 0.25 & 1.2823 & \mathbf{2 . 5 7 E + 0 1} & 1.2823 & \mathbf{2 . 5 7 E + 0 1} \\ \text { "Q_SPRD.3" } & 31.39 & 0.25 & -1.2823 & \mathbf{- 2 . 5 7 E + 0 1} & -1.2823 & \mathbf{- 2 . 5 7 E + 0 1} \\ \text { "Q_SPRD.4" } & 32.44 & 0.25 & 1.2823 & \mathbf{2 . 5 7 E + 0 1} & 1.2823 & \mathbf{2 . 5 7 E + 0 1} \\ \text { "Q_SPRD.5" } & 33.79 & 0.25 & -1.2823 & \mathbf{- 2 . 5 7 E + 0 1} & -1.2823 & \mathbf{- 2 . 5 7 E + 0 1} \\ \text { "Q_SPRD.6" } & 34.84 & 0.25 & 1.2823 & \mathbf{2 . 5 7 E + 0 1} & 1.2823 & \mathbf{2 . 5 7 E + 0 1} \\ \text { "Q_SPRD.7" } & 36.19 & 0.25 & -1.2823 & \mathbf{- 2 . 5 7 E + 0 1} & -1.2823 & \mathbf{- 2 . 5 7 E + 0 1} \\ \text { "Q_SPRD.8" } & 37.24 & 0.25 & 1.2823 & \mathbf{2 . 5 7 E + 0 1} & 1.2823 & \mathbf{2 . 5 7 E + 0 1} \\ \text { "Q_SPRD.9" } & 38.59 & 0.25 & -1.2823 & \mathbf{- 2 . 5 7 E + 0 1} & -1.2823 & \mathbf{- 2 . 5 7 E + 0 1} \\ \text { "Q_SPRD.10" } & 39.64 & 0.25 & 1.2823 & \mathbf{2 . 5 7 E + 0 1} & 1.2823 & \mathbf{2 . 5 7 E + 0 1} \\ \text { "Q_SPRD.11" } & 40.99 & 0.25 & -1.2823 & \mathbf{- 2 . 5 7 E + 0 1} & -1.2823 & \mathbf{- 2 . 5 7 E + 0 1} \\ \text { "Q_SPRD.12" } & 42.04 & 0.25 & 1.2823 & \mathbf{2 . 5 7 E + 0 1} & 1.2823 & \mathbf{2 . 5 7 E + 0 1} \\ \text { "Q_SPRD.7a" } & 43.39 & 0.25 & -0.592772 & \mathbf{- 1 . 1 9 E + 0 1} & -0.592772 & \mathbf{- 1 . 1 9 E + 0 1} \\ \text { "Q_SPRD.8a" } & 44.44 & 0.25 & 0.770314 & \mathbf{1 . 5 4 E + 0 1} & 0.770314 & \mathbf{1 . 5 4 E + 0 1} \\ \text { "Q_SPRD.9a" } & 46.59 & 0.25 & -0.684099 & \mathbf{- 1 . 3 7 E + 0 1} & -0.684099 & \mathbf{- 1 . 3 7 E + 0 1} \\ \text { "Q_SPRD.10a" } & 47.64 & 0.25 & 0.524919 & \mathbf{1 . 0 5 E + 0 1} & 0.524919 & \mathbf{1 . 0 5 E + 0 1}\end{array}$

All bending magnets are rectangular magnets with identical parameters:

Bending angle $=3^{\circ}$ (or $\left.0.05236 \mathrm{rad}\right)$

Magnet length $=0.5 \mathrm{~m}$

\section{Acknowledgements}

The authors thank P. Craievich, M. Veronese and S. Spampinati for useful discussions on the diagnostics and M. Cornacchia and E. Karantzoulis for their guidance regarding beam collimation. This work was partially supported by U.S. DoE contract No: DEAC02-05CH11231.

\section{References}

[1] C. J. Bocchetta et al., "FERMI @ Elettra: A Seeded Harmonic Cascade FEL for EUV and Soft X-Rays", Proc. of the FEL Conf. 2006, Berlin, Germany (August 2006).

[2] P. Emma, R. Brinkmann, SLAC-PUB-7554 (May 1997).

[3] D. Douglas, JLAB-TN-98-012 (March 1998)

[4] F. Löhl, TESLA-FEL 2005-03, Hamburg, Germany (July 2005).

[5] M Cornacchia, et. al., Phys. Rev, Special Topics - Acc. and Beams, 9, (2006)120701.

[6] K. Chow, R. Wells, LBNL-62300 or CBP Note-766 (January 2007).

[7] M. Borland, APS LS-287 (2000). 